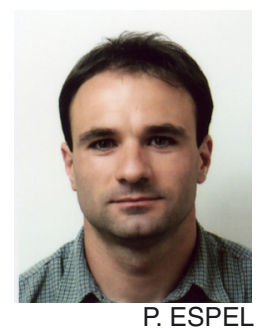

\title{
Nouvelles possibilités d'étalonnage en puissance électrique au LNE
}

\section{New electrical power measurement capabilities at $\mathbf{L N E}$}

\author{
Patrick ESPEL et André POLETAEFF \\ Laboratoire national de métrologie et d'essais (LNE), 29 Avenue Roger Hennequin, 78197 Trappes Cedex, France, patrick.espel@lne.fr.
}

\begin{abstract}
Résumé
L'article présente les nouvelles possibilités d'étalonnage en puissance électrique au LNE. L'étalon primaire est basé sur les techniques d'échantillonnage. Les signaux de tension et de courant sont numérisés puis traités par transformée de Fourier discrète. Jusqu'à présent, cet étalon avait été caractérisé pour les mesures de puissance active en régime sinusoïdal, dans la plage de fréquences $45 \mathrm{~Hz}-65 \mathrm{~Hz}$. Désormais, il permet d'effectuer des raccordements en puissances active et réactive et le domaine de fréquences a été étendu jusqu'à $400 \mathrm{~Hz}$.
\end{abstract}

MOTS CLÉS : WATTMÈTRE NUMÉRIQUE, PUISSANCES ACTIVE ET RÉACTIVE, BILAN D’INCERTITUDE.

\begin{abstract}
The article presents the new electrical power measurement capabilities at LNE. The primary standard is based on sampling techniques. The power is calculated with a discrete Fourier transform algorithm, which is used to process the quantised samples of the voltage and current signals. Up to now, this primary standard was characterised only to measure active power for sinusoidal signals in the $45 \mathrm{~Hz}-65 \mathrm{~Hz}$ frequency range. From now on, the reactive power can also be measured and the frequency range has been extended to $400 \mathrm{~Hz}$.
\end{abstract}

KEY WORDS: DIGITAL WATTMETTER, ACTIVE AND REACTIVE POWERS, UNCERTAINTY BUDGET.

\section{Introduction}

Depuis 2006, le LNE est doté d'une nouvelle référence primaire pour la mesure de la puissance électrique. L'étalon de puissance, basé sur la numérisation des signaux de tension $U$ (de $60 \mathrm{~V}$ à $600 \mathrm{~V}$ ) et de courant $I$ (de $0,1 \mathrm{~A}$ à $100 \mathrm{~A}$ ), permet de mesurer la puissance active $P$, en régime sinusoïdal (ou pour des signaux présentant un taux de distorsion harmonique inférieur à $0,1 \%$ ) et sur la plage de fréquences $45 \mathrm{~Hz}-65 \mathrm{~Hz}$. L'incertitude type relative (par rapport à la puissance apparente) est comprise entre $6 \mu \mathrm{W} / \mathrm{VA}$ et $12 \mu \mathrm{W} / \mathrm{VA}$ pour des courants inférieurs à $10 \mathrm{~A}$ et entre $15 \mu \mathrm{W} / \mathrm{VA}$ et $23 \mu \mathrm{W} / \mathrm{VA}$ pour des courants inférieurs à $100 \mathrm{~A}$.

Pour répondre aux besoins des industriels, les possibilités d'étalonnage en puissance ont été élargies. L'objectif de l'étude est de mesurer les puissances apparente $S$, active $P$ et réactive $Q$ sur la plage de fréquences $45 \mathrm{~Hz}-65 \mathrm{~Hz}$ puis étendre ce domaine aux fréquences industrielles, c'est-à-dire jusqu'à 400 Hz. Un bilan d'incertitude détaillé est présenté pour chacune des puissances. Enfin, la traçabilité des mesures de $U$ et de $I$ aux unités SI est assurée par comparaison avec des convertisseurs thermiques et les mesures de puissance sont validées par comparaison avec un wattmètre commercial récemment étalonné à l'institut national allemand PhysikalischTechnische Bundesanstalt (PTB).

\section{Rappel sur le principe de fonctionnement du wattmètre étalon}

Une source de puissance fictive délivre des signaux sinusoïdaux de tension $u(t)$ et de courant $i(t)$ sur deux circuits différents. Le principe du wattmètre numérique consiste à échantillonner simultanément ces signaux, à des intervalles de temps égaux et pendant un nombre entier de périodes (fig. 1). Cette opération est réalisée par deux voltmètres numériques qui, pour une plus grande précision des mesures, sont utilisés sur le calibre $1 \mathrm{~V}$, en mode échantillonnage par lecture de tension continue (mode DCV). Les signaux $u(t)$ et $i(t)$ sont au préalable 


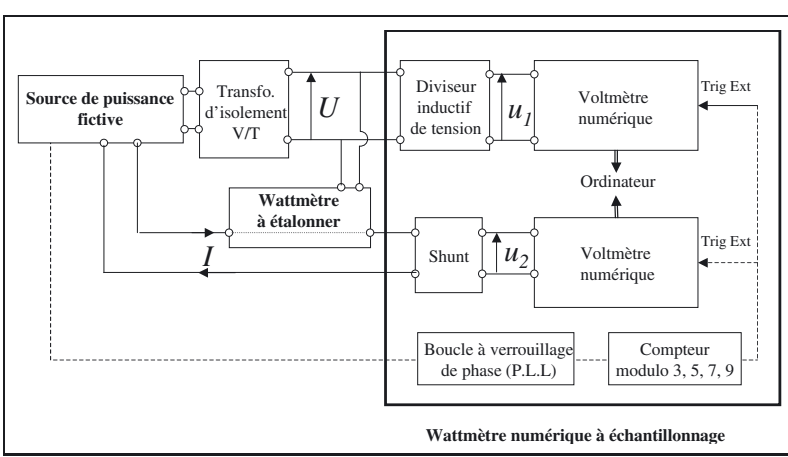

Fig. 1. - Schéma du montage de l'étalonnage d'un wattmètre commercial à partir du wattmètre primaire.

mis en forme respectivement par l'intermédiaire d'un diviseur inductif de tension et d'un shunt de courant. On obtient ainsi deux signaux $u_{1}(t)$ et $u_{2}(t)$. Notons qu'un transformateur d'isolement est placé dans le circuit de tension afin d'éviter le passage d'un courant continu qui pourrait entraîner la saturation des tores magnétiques du diviseur inductif.

Lors de la numérisation des signaux $u_{1}(t)$ et $u_{2}(t)$, on prélève $N$ échantillons sur $M$ périodes. Ces échantillons $x\left(k T_{\mathrm{e}}\right)$ sont ensuite analysés par transformée de Fourier discrète $(\mathrm{TFd})$.

La décomposition des fonctions $u(t)=U \sin (2 \pi f+$ $\left.\phi_{\mathrm{u}}\right)$ et $i(t)=I \sin \left(2 \pi f+\phi_{\mathrm{i}}\right)$ en série de Fourier permet la détermination des valeurs efficaces $U$ et $I$ de la tension et du courant et du déphasage $\phi=\phi_{\mathrm{i}}-\phi_{\mathrm{u}}$ ainsi que le calcul des puissances apparente $S$, active $P$ et réactive $Q$, à partir des formules suivantes :

$$
\begin{aligned}
& S=U I \\
& P=U I \cdot \cos \phi \\
& Q=U I \sin \phi .
\end{aligned}
$$

L'une des principales difficultés de cette méthode consiste à prélever un nombre entier de périodes des signaux afin d'éviter des erreurs de troncature. Pour cela, les voltmètres sont déclenchés par un signal de cadencement généré par une boucle de réaction à verrouillage de phase (PLL) qui permet de synchroniser la fréquence $f$ des signaux et la fréquence d'échantillonnage $f_{\mathrm{e}}$ :

$$
f_{\mathrm{e}}=\frac{N}{M} \cdot f \text {. }
$$

\section{Extension des mesures de tension et de courant aux fréquences industrielles}

La première étape consiste à caractériser les convertisseurs analogique-numérique (CAN) des voltmètres sur le calibre $1 \mathrm{~V}$, pour des signaux continus (caractérisation statique) et des signaux alternatifs jusqu'aux fréquences industrielles (caractérisation dynamique). Ensuite, afin de mesurer des tensions $U$ ou des courants $I$ supérieurs sur ce même calibre, il faudra insérer dans le montage un diviseur de tension et un shunt.

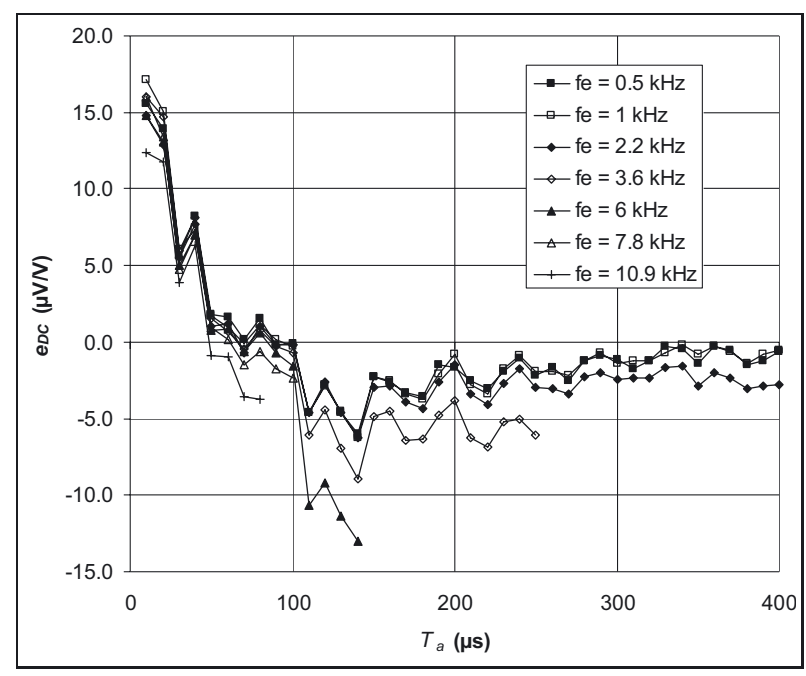

Fig. 2. - Erreur $e_{\mathrm{DC}}$ en fonction du temps d'intégration $T_{\mathrm{a}}$ des CAN, pour différentes fréquences d'échantillonnage $f_{\mathrm{e}}$.

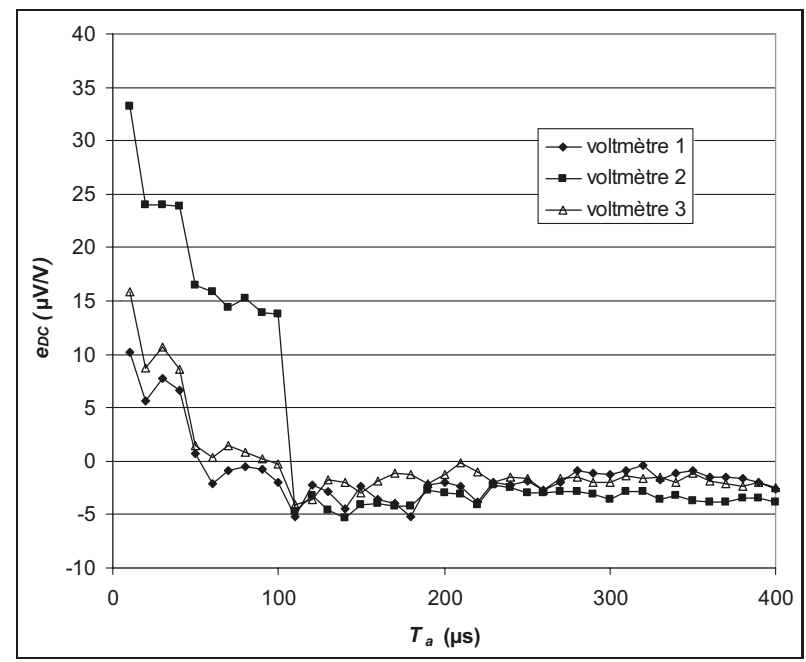

Fig. 3. - Erreur $e_{\mathrm{DC}}$ en fonction du temps d'intégration $T_{\mathrm{a}}$ des CAN, pour différents voltmètres et $f_{\mathrm{e}}=2,2 \mathrm{kHz}$.

\subsection{Caractérisations des CAN sur le calibre 1 V}

\subsubsection{Caractérisation statique}

Les voltmètres sont utilisés en mode DCV, c'està-dire en mode échantillonnage par lecture de tension continue. Il est donc nécessaire, avant toute mesure en alternatif, de caractériser les voltmètres sous tension continue et de déterminer l'erreur $e_{\mathrm{DC}}$, différence entre la tension lue par le voltmètre et la tension de référence appliquée. Tous les paramètres d'influence (temps d'intégration, fréquence d'échantillonnage, temps mort...) ont été étudiés [1-4]. Quelques résultats sont présentés dans les figures 2 et 3 .

La figure 2 montre que l'erreur $e_{\mathrm{DC}}$ du voltmètre dépend fortement du temps d'intégration $T_{\mathrm{a}}$ des CAN et de la fréquence d'échantillonnage $f_{\mathrm{e}}$. La figure 3 montre que l'allure de la caractéristique $e_{\mathrm{DC}}=g\left(T_{\mathrm{a}}\right)$ est la même pour tous les voltmètres testés avec toutefois de 


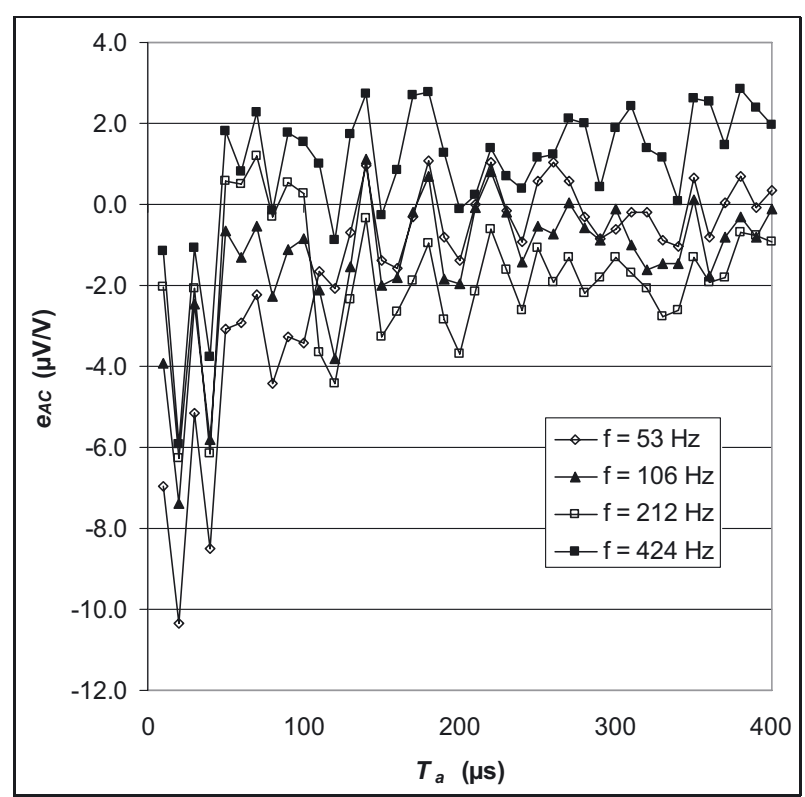

Fig. 4. - Erreur $e_{\mathrm{AC}}$ pour un signal de valeur efficace $0,8 \mathrm{~V}$ et de fréquence $f$ en fonction du temps d'intégration $T_{\mathrm{a}}$ des CAN.

fortes variations en amplitude pour des temps d'intégration courts, c'est-à-dire inférieurs à $100 \mu \mathrm{s}$.

À partir des résultats expérimentaux, on déduit que, pour des temps d'intégration suffisamment longs (typiquement supérieurs à $150 \mu$ s et inférieurs à $400 \mu$ s) et des fréquences d'échantillonnage inférieures à $3 \mathrm{kHz}$, la valeur moyenne de l'erreur $e_{\mathrm{DC}}$ des différents voltmètres est égale à $-3 \mu \mathrm{V} / \mathrm{V}$. L'incertitude type relative associée est inférieure à $2 \mu \mathrm{V} / \mathrm{V}$.

\subsubsection{Caractérisation dynamique}

La caractérisation dynamique a été effectuée en comparant les mesures de la valeur efficace des signaux par les techniques d'échantillonnage aux mesures effectuées au moyen de convertisseurs thermiques [2]. On a défini l'erreur $e_{\mathrm{AC}}$ comme la différence relative entre les valeurs mesurées par les deux techniques.

Lors de l'opération de numérisation avec le voltmètre, $N$ échantillons sont prélevés sur 25 périodes avec une fréquence d'échantillonnage de 2,2 kHz. Cela permet de respecter le théorème de Shannon (qui impose que la fréquence d'échantillonnage soit au moins deux fois supérieure à la fréquence maximale contenue dans le signal) pour toute la gamme de fréquences considérées tout en ayant la possibilité de choisir des temps d'intégration relativement longs (jusqu'à $400 \mu \mathrm{s}$ ). La figure 4 présente les résultats de la comparaison et montre que l'erreur $e_{\mathrm{AC}}$, pour un signal dont la valeur efficace est de $0,8 \mathrm{~V}$, dépend fortement du temps d'intégration $T_{\mathrm{a}}$ des CAN particulièrement pour les faibles valeurs de $T_{\mathrm{a}}$.

L'allure générale de la caractéristique $e_{\mathrm{AC}}=g\left(T_{\mathrm{a}}\right)$ reste la même pour les différentes valeurs de la fréquence $f$ des signaux étudiés. Seules, les valeurs de l'erreur relative diffèrent de quelques microvolts par volt. De façon générale, pour des $T_{\mathrm{a}}$ supérieurs à $150 \mu \mathrm{s}$, l'erreur $e_{\mathrm{AC}}$ n'excède pas $3 \mu \mathrm{V} / \mathrm{V}$ sur toute la plage des fréquences considérées.

Par la suite, les signaux seront échantillonnés avec une fréquence $f_{\mathrm{e}}$ de $2,2 \mathrm{kHz}$ et quantifiés avec un temps d'intégration de $200 \mu$ s.

\subsubsection{Principales sources d'erreurs et bilan d'incertitude}

Les mesures des grandeurs efficaces $u_{1}$ et $u_{2}$ des signaux sont affectées par des erreurs dues à la résolution des voltmètres $\varepsilon_{\text {res }}$, à leur limitation de bande passante $\varepsilon_{\mathrm{BP}}$, au temps d'intégration des convertisseurs $\varepsilon_{\mathrm{T}}$, à la quantification $\varepsilon_{\mathrm{Q}}$ et aux fluctuations de la période d'échantillonnage $\varepsilon_{\mathrm{j}}$. D'autre part, les voltmètres étant utilisés en mode DCV, ils sont préalablement étalonnés à partir d'une source continue de référence. Une erreur $\varepsilon_{\mathrm{DC}}$ vient donc s'ajouter aux erreurs précédemment citées. Les termes $u_{1}$ et $u_{2}$ peuvent donc s'écrire sous la forme de la relation (5):

$$
u_{1}=u_{2}=u_{\text {lue }}\left(1+\varepsilon_{\text {res }}+\varepsilon_{\mathrm{BP}}+\varepsilon_{\mathrm{T}}+\varepsilon_{\mathrm{Q}}+\varepsilon_{\mathrm{j}}+\varepsilon_{\mathrm{DC}}\right)
$$

où $\varepsilon_{\text {res }}, \varepsilon_{\mathrm{BP}}, \varepsilon_{\mathrm{T}}, \varepsilon_{\mathrm{Q}}$, $\varepsilon_{\mathrm{j}}$ et $\varepsilon_{\mathrm{DC}}$ sont des erreurs relatives.

En appliquant la loi de propagation des incertitudes à la relation (5), on obtient l'expression (6) :

$\left(\frac{\sigma_{\mathrm{u}_{1,2}}}{u_{1,2}}\right)^{2}=\left(\frac{\sigma_{\mathrm{u}_{\mathrm{lue}}}}{u_{\text {lue }}}\right)^{2}+\frac{\sigma_{\mathrm{res}}^{2}+\sigma_{\mathrm{BP}}^{2}+\sigma_{\mathrm{T}}^{2}+\sigma_{\mathrm{Q}}^{2}+\sigma_{\mathrm{j}}^{2}+\sigma_{\mathrm{DC}}^{2}}{\left(1+\varepsilon_{\mathrm{res}}+\varepsilon_{\mathrm{BP}}+\varepsilon_{\mathrm{T}}+\varepsilon_{\mathrm{Q}}+\varepsilon_{\mathrm{j}}+\varepsilon_{\mathrm{DC}}\right)^{2}}$.

Les différentes sources d'erreurs sont détaillées :

- la première composante d'incertitude $\sigma_{\text {lue }}$ est liée à la dispersion de la mesure. L'évaluation de type A de cette composante se fait par la mesure de l'écart type. L'incertitude type relative est inférieure à $3 \times 10^{-6}$ pour des séries de 20 mesures.

- la composante $\sigma_{\text {res }}$ est liée à la résolution du voltmètre. En supposant que la loi de probabilité de l'erreur associée est rectangulaire, l'incertitude type relative (évaluation de type B) est inférieure à $4 \times 10^{-7}$, pour un temps d'intégration de $200 \mu \mathrm{s}$.

- l'erreur $\varepsilon_{\mathrm{BP}}$ liée à la limitation de bande passante des voltmètres.

Sur le calibre $1 \mathrm{~V}$, la bande passante des voltmètres est limitée à $150 \mathrm{kHz}$. En modélisant cette bande passante par un filtre passe-bas du deuxième ordre [3], l'erreur relative $\varepsilon_{\mathrm{BP}}$ peut s'écrire sous la forme :

$$
\varepsilon_{\mathrm{BP}}=\left(\frac{\sigma_{\mathrm{u}_{1}}}{u_{1}}\right)_{\mathrm{BP}}=\left(\frac{\sigma_{\mathrm{u}_{2}}}{u_{2}}\right)_{\mathrm{BP}}=\sqrt{\frac{1}{1+7(R C \omega)^{2}+(R C \omega)^{4}}}-1 .
$$

Aux fréquences industrielles, cette expression peut être simplifiée et s'écrire sous la forme :

$$
\varepsilon_{\mathrm{BP}}=\frac{1}{\sqrt{1+7(R C \omega)^{2}}}-1 .
$$


Tableau 1

Erreur de bande passante $\varepsilon_{\mathrm{BP}}$ en fonction de la valeur de la fréquence $f$.

\begin{tabular}{|c|c|c|c|c|}
\hline$f(\mathrm{~Hz})$ & 53 & 100 & 200 & 400 \\
\hline$\varepsilon_{\mathrm{BP}}$ & $-6,2 \times 10^{-8}$ & $-2,3 \times 10^{-7}$ & $-9,3 \times 10^{-7}$ & $-3,7 \times 10^{-6}$ \\
\hline
\end{tabular}

Tableau 2

$\varepsilon_{\mathrm{T}}=g(f)$ et $\sigma_{\mathrm{T}}=g(f)$ pour $T_{\mathrm{a}}=200 \mu \mathrm{s}$.

\begin{tabular}{|c|c|c|c|c|}
\hline$f(\mathrm{~Hz})$ & 53 & 100 & 200 & 400 \\
\hline$\varepsilon_{\mathrm{T}}\left(\times 10^{-6}\right)$ & -185 & -658 & -2630 & -10494 \\
\hline$\sigma_{\mathrm{T}}\left(\times 10^{-6}\right)$ & 0,07 & 0,2 & 0,9 & 3,7 \\
\hline
\end{tabular}

L'erreur $\varepsilon_{\mathrm{BP}}$ est calculée en fonction de la valeur de la fréquence $f$ des signaux étudiés (tab. 1).

L'incertitude type relative associée (évaluation de type B) est négligeable.

- l'erreur liée au temps d'intégration.

En pratique, l'opération d'échantillonnage n'est pas réalisée avec une impulsion infiniment brève. Le voltmètre établit une moyenne sur le signal d'entrée pendant une durée $T_{\mathrm{a}}$ qui correspond au temps d'intégration. L'erreur relative résultante $\varepsilon_{\mathrm{T}}$ sur la mesure de la tension efficace est alors :

$$
\varepsilon_{\mathrm{T}}=\left(\frac{\sigma_{\mathrm{u}_{1,2}}}{u_{1,2}}\right)_{\mathrm{Ta}}=\frac{\sin \left(\pi f T_{\mathrm{a}}\right)}{\pi f T_{\mathrm{a}}}-1 .
$$

L'erreur $\varepsilon_{\mathrm{T}}$ et son incertitude type relative associée $\sigma_{\mathrm{T}}$ (évaluation de type $\mathrm{B}$ déterminée en appliquant la loi de propagation des incertitudes à la relation (9) et en supposant que la loi de probabilité de l'erreur associée est rectangulaire) sont calculées en fonction de la valeur de la fréquence $f$ des signaux étudiés (tab. 2), pour un temps d'intégration de $200 \mu$ s.

- l'erreur $\varepsilon_{\mathrm{Q}}$ liée à la quantification du signal.

Lors de la phase de quantification, il y a dégradation de l'information. En effet, le signal quantifié a subi une distorsion harmonique, du fait de la non-linéarité de la fonction de transfert du quantificateur (fonction en marche d'escalier). On peut donc considérer que le signal quantifié $s_{\mathrm{q}}$ est la somme d'un signal analogique $s$ et d'un bruit $\varepsilon_{\mathrm{Q}}$ (bruit ou erreur de quantification) de valeur moyenne nulle :

$$
s_{\mathrm{q}}(m)=s(m)+\varepsilon_{\mathrm{Q}}(m), \text { pour le } m^{\mathrm{e}} \text { point. }
$$

En supposant que la loi de probabilité de l'erreur associée est rectangulaire et que la valeur efficace du signal soit calculée à partir de la TFd de $N$ échantillons, l'incertitude type sur le bruit de quantification est donnée par la relation :

$$
\sigma_{\mathrm{Q}}=\frac{\Delta}{\sqrt{6 N}}
$$

où $\Delta$ est le pas de quantification.
Tableau 3

$\sigma_{\mathrm{Q}}=g(f)$ pour $T_{\mathrm{a}}=200 \mu \mathrm{s}, f_{\mathrm{e}}=2,2 \mathrm{kHz}$ et $M=25$.

\begin{tabular}{|c|c|c|c|c|}
\hline$f(\mathrm{~Hz})$ & 50 & 100 & 200 & 400 \\
\hline$\sigma_{\mathrm{Q}}$ & $1,2 \times 10^{-8}$ & $1,7 \times 10^{-8}$ & $2,4 \times 10^{-8}$ & $3,4 \times 10^{-8}$ \\
\hline
\end{tabular}

Tableau 4

Incertitude type relative sur la mesure de la valeur efficace des tensions $u_{1}(t)$ et $u_{2}(t)$.

\begin{tabular}{|c|c|c|c|c|}
\hline$f(\mathrm{~Hz})$ & 53 & 100 & 200 & 400 \\
\hline$\frac{\sigma_{u_{1,2}}}{u_{1,2}}\left(\times 10^{-6}\right)$ & 3,2 & 3,2 & 3,3 & 4,9 \\
\hline
\end{tabular}

Si le codage du signal se fait sur $n$ bits ( $n$ dépendant du temps d'intégration $T_{\mathrm{a}}$ ) et le voltmètre est utilisé sur le plein calibre $1 \mathrm{~V}$, ce pas de quantification $\Delta$ est égal à :

$$
\Delta=\frac{2}{2^{n}}
$$

Le bruit ou erreur de quantification est d'autant plus faible que le pas de quantification $\Delta$ est petit.

L'incertitude type $\sigma_{\mathrm{Q}}$ est calculée en fonction de la valeur de la fréquence $f$ des signaux étudiés (tab. 3), pour un temps d'intégration de $200 \mu \mathrm{s}$ ( $n=18$ bits).

- l'erreur $\varepsilon_{\mathrm{j}}$ liée aux fluctuations de la période d'échantillonnage.

La fluctuation de la période d'échantillonnage peut entraîner des erreurs sur les mesures de la valeur efficace des signaux $u_{1}(t)$ et $u_{2}(t)$. Des simulations réalisées sous Labview montrent que la contribution du jitter d'échantillonnage est négligeable compte tenu des incertitudes recherchées aux fréquences industrielles.

- erreur $\varepsilon_{\mathrm{DC}}$ des voltmètres.

Les tests réalisés ont montré que l'incertitude type relative associée à la mesure d'une tension continue échantillonnée à $2,2 \mathrm{kHz}$ et quantifiée avec un temps d'intégration de $200 \mu$ s est de l'ordre de $1 \times 10^{-6}$.

Tous les termes d'erreurs et leurs incertitudes associées ont été évalués. La plupart d'entre eux sont négligeables. L'expression (6) peut donc être simplifiée et s'écrire sous la forme :

$$
\left(\frac{\sigma_{\mathrm{u}_{1,2}}}{u_{1,2}}\right)^{2} \approx\left(\frac{\sigma_{\mathrm{u}_{\text {lue }}}}{u_{\text {lue }}}\right)^{2}+\sigma_{\mathrm{DC}}^{2}+\sigma_{\mathrm{T}}^{2}
$$

En combinant toutes les sources d'incertitude, l'incertitude type relative sur la mesure de la valeur efficace d'une tension sinusoïdale est inférieure à $4,9 \mu \mathrm{V} / \mathrm{V}$ sur la plage de fréquences de $50 \mathrm{~Hz}$ à $400 \mathrm{~Hz}$ (tab. 4).

\subsection{Mesures de tension et de courant en régime} sinusoïdal, aux fréquences industrielles

\subsubsection{Mesures de tension}

Les mesures de tension $U$ se font en associant le voltmètre (utilisé sur le calibre $1 \mathrm{~V}$ ) et un diviseur inductif de 
Tableau 5

Incertitude-type relative sur le rapport de transformation $k \mathrm{du}$ diviseur inductif.

\begin{tabular}{|c|c|}
\hline$k_{\text {nominal }}$ & $\sigma_{k} / k$ \\
\hline 100 & $5,9 \times 10^{-9}$ \\
\hline 111,11111 & $6,6 \times 10^{-9}$ \\
\hline 125 & $7,4 \times 10^{-9}$ \\
\hline 142,857143 & $8,4 \times 10^{-9}$ \\
\hline 166,666667 & $9,8 \times 10^{-9}$ \\
\hline 200 & $1,2 \times 10^{-8}$ \\
\hline 250 & $1,5 \times 10^{-8}$ \\
\hline 333,333333 & $2,0 \times 10^{-8}$ \\
\hline 500 & $3,0 \times 10^{-8}$ \\
\hline 1000 & $5,9 \times 10^{-8}$ \\
\hline
\end{tabular}

tension (de rapport de transformation $k$ ). Les valeurs de $U$ sont alors déterminées à partir de l'équation suivante :

$$
U=k \cdot u_{1}
$$

En appliquant la loi de propagation des incertitudes à l'équation (14), l'incertitude type relative sur la mesure de $U$ se déduit de :

$$
\left(\frac{\sigma_{U}}{U}\right)^{2}=\left(\frac{\sigma_{\mathrm{u}_{1}}}{u_{1}}\right)^{2}+\left(\frac{\sigma_{k}}{k}\right)^{2}
$$

Le diviseur utilisé a été fabriqué au National Measurement Institute of Australia (NMIA). Il fonctionne jusqu'à une fréquence de $1 \mathrm{kHz}$ (sans introduire de distorsion harmonique significative sur le signal) et la tension maximale d'entrée est de $1000 \mathrm{~V}$. Le rapport de transformation a été déterminé sous $100 \mathrm{~V}, 800 \mathrm{~V}$ et $1000 \mathrm{~V}$ à des fréquences de $40 \mathrm{~Hz}, 63 \mathrm{~Hz}, 400 \mathrm{~Hz}$ et $1 \mathrm{kHz}$. L'incertitude type relative associée à $k$ est toujours inférieure à $5,9 \times 10^{-8}$ (tab. 5).

Pour connaître les valeurs du rapport de transformation $k$ à des fréquences différentes de celles fournies par le certificat d'étalonnage, une interpolation est nécessaire. Cette interpolation n'introduit pas d'incertitudes significatives supplémentaires sur la mesure de la tension.

Compte-tenu des résultats présentés dans le tableau 5, le deuxième terme de l'équation (15) est négligeable. On en déduit alors que l'incertitude type relative sur la mesure de $U$ est égale à celle de $u_{1}$. Autrement dit, si la tension $u_{1}$ est égale à $0,8 \mathrm{~V}$, l'incertitude type relative est inférieure à $5 \mu \mathrm{V} / \mathrm{V}$ de $50 \mathrm{~Hz}$ à $400 \mathrm{~Hz}$. Elle reste inchangée tant que la valeur efficace de $u_{1}$ est comprise entre $0,45 \mathrm{~V}$ et $0,9 \mathrm{~V}$. Si le rapport de transformation du diviseur inductif est choisi de sorte que la valeur efficace de $u_{1}$ soit inférieure à $0,45 \mathrm{~V}$, les mesures présentent plus de dispersion et l'incertitude est dégradée (supérieure à $10 \mu \mathrm{V} / \mathrm{V})$.

\subsubsection{Mesures de courant}

Le courant $I$ est mesuré par l'intermédiaire d'un shunt de courant d'impédance $Z$ :

$$
I=\frac{u_{2}}{|Z|} \text { avec }|Z|=\frac{R}{(1+\delta)}
$$

où $|Z|$ est le module de l'impédance du shunt de courant, $R$ est la valeur de sa résistance en continu et $\delta$ est son écart de transposition ou différence relative entre la valeur du module de l'impédance en régime alternatif et la valeur de sa résistance en régime continu.

En appliquant la loi de propagation des incertitudes à l'équation (16), l'incertitude type relative sur la mesure de I s'écrit :

$$
\left(\frac{\sigma_{I}}{I}\right)^{2}=\left[\left(\frac{\sigma_{R}}{R}\right)^{2}+\left(\frac{\sigma_{\delta}}{1+\delta}\right)^{2}\right]+\left(\frac{\sigma_{\mathrm{u}_{2}}}{u_{2}}\right)^{2} .
$$

Les différents shunts de courant utilisés ont été étalonnés à l'institut national suédois Swedish National Testing and Research Institute (SP). Les valeurs du courant maximal admis $I$, des résistances $R$ et des écarts de transposition $\delta$ sont présentés dans les tableaux 6 et 7 avec leurs incertitudes types associées.

L'incertitude type relative sur la mesure de $I$ est calculée à partir de la formule 17 en tenant compte des valeurs présentées dans les tableaux 6 et 7. Sur la gamme des fréquences considérées, elle est toujours inférieure à $21 \mu \mathrm{A} / \mathrm{A}$ jusqu'à $100 \mathrm{~A}$. Pour les plus faibles courants (jusqu'à $10 \mathrm{~A}$ ), elle reste inférieure à $12 \mu \mathrm{A} / \mathrm{A}$.

\section{Applications aux mesures de puissance}

Les puissances apparente $S$, active $P$ et réactive $Q$ sont calculées à partir des relations (1), (2) et (3). En appliquant la loi de propagation des incertitudes à ces relations, on obtient une expression qui fait apparaître des termes d'incertitudes en phase et en quadrature :

$$
\begin{aligned}
&\left(\frac{\sigma_{\mathrm{S}}}{S}\right)^{2}=\underbrace{\left(\frac{\sigma_{U}}{U}\right)^{2}+\left(\frac{\sigma_{I}}{I}\right)^{2}}_{\begin{array}{l}
\text { Incertitudes } \\
\text { en phase }
\end{array}} \\
&\left(\frac{\sigma_{P}}{P}\right)^{2}=\underbrace{\left(\frac{\sigma_{U}}{U}\right)^{2}+\left(\frac{\sigma_{I}}{I}\right)^{2}}_{\begin{array}{l}
\text { Incertitudes } \\
\text { en phase }
\end{array}}+\underbrace{\tan ^{2} \phi \cdot \sigma_{\phi}^{2}}_{\begin{array}{l}
\text { Incertitudes } \\
\text { en quadrature }
\end{array}} \\
&\left(\frac{\sigma_{\mathrm{Q}}}{Q}\right)^{2}=\underbrace{\left(\frac{\sigma_{U}}{U}\right)^{2}+\left(\frac{\sigma_{I}}{I}\right)^{2}}_{\begin{array}{l}
\text { Incertitudes } \\
\text { en phase }
\end{array}}+\underbrace{\frac{1}{\tan ^{2} \phi} \cdot \sigma_{\phi}^{2}}_{\begin{array}{l}
\text { Incertitudes } \\
\text { enquadrature }
\end{array}} .
\end{aligned}
$$

Dans l'expression (19), le terme d'incertitude en quadrature a une contribution nulle sur le bilan global pour 
Tableau 6

Valeurs des résistances des différents shunts de courant, en régime continu, à $50 \%$ et $100 \%$ du courant nominal $I_{N}$.

\begin{tabular}{|c|c|c|}
\hline Courant maximal admis & $R\left(1 \pm \sigma_{R} / R\right)\left(50 \%\right.$ de $\left.I_{\mathrm{n}}\right)$ & $R\left(1 \pm \sigma_{R} / R\right)\left(100 \%\right.$ de $\left.I_{\mathrm{n}}\right)$ \\
\hline $0,1 \mathrm{~A}$ & $7,999334 \times\left(1 \pm 0,5 \times 10^{-6}\right) \Omega$ & $7,999311 \times\left(1 \pm 0,5 \times 10^{-6}\right) \Omega$ \\
\hline $0,5 \mathrm{~A}$ & $1,599932 \times\left(1 \pm 1,5 \times 10^{-6}\right) \Omega$ & $1,599928 \times\left(1 \pm 1 \times 10^{-6}\right) \Omega$ \\
\hline $1 \mathrm{~A}$ & $0,799984 \times\left(1 \pm 1 \times 10^{-6}\right) \Omega$ & $0,799983 \times\left(1 \pm 1 \times 10^{-6}\right) \Omega$ \\
\hline $2 \mathrm{~A}$ & $399,9948 \times\left(1 \pm 3 \times 10^{-6}\right) \mathrm{m} \Omega$ & $399,9943 \times\left(1 \pm 2 \times 10^{-6}\right) \mathrm{m} \Omega$ \\
\hline $5 \mathrm{~A}$ & $160,0155 \times\left(1 \pm 2 \times 10^{-6}\right) \mathrm{m} \Omega$ & $160,0158 \times\left(1 \pm 1 \times 10^{-6}\right) \mathrm{m} \Omega$ \\
\hline $10 \mathrm{~A}$ & $79,98845 \times\left(1 \pm 2 \times 10^{-6}\right) \mathrm{m} \Omega$ & $79,98672 \times\left(1 \pm 1 \times 10^{-6}\right) \mathrm{m} \Omega$ \\
\hline $100 \mathrm{~A}$ & $7,99453 \times\left(1 \pm 2 \times 10^{-6}\right) \mathrm{m} \Omega$ & $7,99332 \times\left(1 \pm 2 \times 10^{-6}\right) \mathrm{m} \Omega$ \\
\hline
\end{tabular}

Tableau 7

Ecart de transposition $\delta(\mu \mathrm{A} / \mathrm{A})$ et incertitude-type associée en fonction de la fréquence $f$ du signal, pour les différents shunts de courant.

\begin{tabular}{|c|c|c|c|c|}
\hline$f(\mathrm{~Hz})$ & 20 & 45 & 110 & 400 \\
\hline $0,1 \mathrm{~A}$ & $4 \pm 10$ & $2 \pm 10$ & $0 \pm 10$ & $0 \pm 10$ \\
\hline $0,5 \mathrm{~A}$ & $1 \pm 12,5$ & $3 \pm 10$ & $0 \pm 10$ & $1 \pm 10$ \\
\hline $1 \mathrm{~A}$ & $0 \pm 12,5$ & $2 \pm 10$ & $1 \pm 10$ & $-1 \pm 10$ \\
\hline $2 \mathrm{~A}$ & $1 \pm 12,5$ & $1 \pm 10$ & $1 \pm 10$ & $0 \pm 10$ \\
\hline 5 A & $1 \pm 15$ & $0 \pm 10$ & $-1 \pm 10$ & $-1 \pm 10$ \\
\hline $10 \mathrm{~A}$ & $0 \pm 15$ & $0 \pm 12,5$ & $0 \pm 12,5$ & $0 \pm 12,5$ \\
\hline $100 \mathrm{~A}$ & $1 \pm 22,5$ & $0 \pm 20$ & $1 \pm 20$ & $0 \pm 20$ \\
\hline
\end{tabular}

$\phi=0^{\circ}$ et une contribution qui tend vers l'infini lorsque $\phi$ tend vers $90^{\circ}$. Dans l'expression (20), c'est l'inverse. Pour s'affranchir de ce problème, les incertitudes types relatives sur les mesures des puissances active et réactive s'exprimeront toujours en fonction de la puissance apparente (21), (22) :

$$
\begin{aligned}
& \left(\frac{\sigma_{P}}{S}\right)^{2}=\left[\left(\frac{\sigma_{U}}{U}\right)^{2}+\left(\frac{\sigma_{I}}{I}\right)^{2}\right] \cos ^{2} \phi+\sin ^{2} \phi \cdot \sigma_{\phi}^{2} \\
& \left(\frac{\sigma_{Q}}{S}\right)^{2}=\left[\left(\frac{\sigma_{U}}{U}\right)^{2}+\left(\frac{\sigma_{I}}{I}\right)^{2}\right] \sin ^{2} \phi+\cos ^{2} \phi \cdot \sigma_{\phi}^{2} .
\end{aligned}
$$

Les termes $\left(\sigma_{U} / U\right)$ et $\left(\sigma_{I} / I\right)$ sont connus jusqu'à $400 \mathrm{~Hz}$. La première étape consiste donc à analyser le déphasage $\phi$ entre la tension $u(t)$ et le courant $i(t)$. Ensuite, des bilans d'incertitude sont établis pour chacune des puissances. Enfin, la plupart des mesures sont validées par une comparaison avec un wattmètre commercial préalablement étalonné à la PTB.

\subsection{Analyse des différentes sources de déphasage}

Les erreurs de déphasage sont introduites par le diviseur inductif $\phi_{\mathrm{IVD}}$, le shunt de courant $\phi_{\mathrm{s}}$ et les voltmètres $\phi_{\mathrm{v}}$ pour lesquels on distingue les déphasages dues à la différence de bande passante $\phi_{\mathrm{BP}}$, à la différence de
Tableau 8

Erreurs de déphasage $\phi_{\mathrm{IVD}}$ du diviseur inductif de tension à $53 \mathrm{~Hz}$ et $400 \mathrm{~Hz}$, sous $1000 \mathrm{~V}$.

\begin{tabular}{|c|c|c|}
\hline & \multicolumn{2}{|c|}{$\phi_{\text {IVD }}(\mu \mathrm{rad})$} \\
\hline$k_{\text {nominal }}$ & $f=53 \mathrm{~Hz}$ & $f=400 \mathrm{~Hz}$ \\
\hline 100 & 1,1 & 6,9 \\
\hline 111,11 & 1,1 & 7,0 \\
\hline 125 & 1,2 & 7,3 \\
\hline 142,85 & 1,2 & 7,5 \\
\hline 166,66 & 1,2 & 7,7 \\
\hline 200 & 1,3 & 7,8 \\
\hline 250 & 1,3 & 8,0 \\
\hline 333,33 & 1,3 & 8,3 \\
\hline 500 & 1,4 & 8,5 \\
\hline 1000 & 1,4 & 9,0 \\
\hline
\end{tabular}

temps d'intégration $\phi_{\mathrm{T}}$, au retard de déclenchement $\phi_{\mathrm{r}}$ et aux fluctuations de la période d'échantillonnage $\phi_{\mathrm{j}}$ :

$$
\phi=\phi_{\mathrm{o}}+\phi_{\mathrm{IVD}}+\phi_{\mathrm{s}}+\phi_{\mathrm{v}}
$$

avec $\phi_{\mathrm{v}}=\phi_{\mathrm{BP}}+\phi_{\mathrm{T}}+\phi_{\mathrm{r}}+\phi_{\mathrm{j}}$ et $\phi_{0}$ est le déphasage initial (au niveau de la source de puissance) entre $u(t)$ et $i(t)$.

L'incertitude type associée s'écrit alors :

$$
\sigma_{\phi}^{2}=\sigma_{\phi_{I V D}}^{2}+\sigma_{\phi_{\mathrm{s}}}^{2}+\sigma_{\phi_{\mathrm{v}}}^{2} \text {. }
$$

Tous les termes d'erreurs et leurs incertitudes associées ont été évalués expérimentalement et/ou par le calcul :

- les erreurs de déphasage $\phi_{I V D}$ du diviseur inductif sont présentées dans le tableau 8, pour des signaux d'amplitude $1000 \mathrm{~V}$ et de fréquences $53 \mathrm{~Hz}$ et $400 \mathrm{~Hz}$. Ces erreurs restent inchangées pour tous les niveaux de tension compris entre $60 \mathrm{~V}$ et $1000 \mathrm{~V}$. Les incertitudes types associées n'excèdent pas $0,3 \mu \mathrm{rad}$.

- les erreurs de déphasage $\phi_{\mathrm{S}}$ des différents shunts sont présentées dans le tableau 9, pour des signaux de fréquences $50 \mathrm{~Hz}$ et $400 \mathrm{~Hz}$. 
Tableau 9

Erreurs de déphasage $\phi_{\mathrm{s}}$ et incertitudes types associées pour les différents shunts de courant, à $50 \mathrm{~Hz}$ et $400 \mathrm{~Hz}$.

\begin{tabular}{|c|c|c|c|c|}
\hline & \multicolumn{2}{|c|}{$f=50 \mathrm{~Hz}$} & \multicolumn{2}{c|}{$f=400 \mathrm{~Hz}$} \\
\hline Shunt & $\phi_{\mathrm{s}}(\mu \mathrm{rad})$ & $\sigma_{\phi \mathrm{s}}(\mu \mathrm{rad})$ & $\phi_{\mathrm{s}}(\mu \mathrm{rad})$ & $\sigma_{\phi \mathrm{s}}(\mu \mathrm{rad})$ \\
\hline $0,1 \mathrm{~A}$ & 0,1 & $\pm 0,5$ & 1,2 & \pm 4 \\
\hline $0,5 \mathrm{~A}$ & 0,1 & $\pm 0,4$ & 0,7 & \pm 3 \\
\hline $1 \mathrm{~A}$ & 0,1 & $\pm 0,4$ & 1,0 & \pm 3 \\
\hline $2 \mathrm{~A}$ & 0,0 & $\pm 0,4$ & $-0,2$ & \pm 3 \\
\hline $5 \mathrm{~A}$ & 0,4 & $\pm 0,6$ & 2,9 & $\pm 4,5$ \\
\hline $10 \mathrm{~A}$ & 1,1 & \pm 1 & 8 & \pm 8 \\
\hline $100 \mathrm{~A}$ & 1,4 & \pm 10 & 11 & \pm 40 \\
\hline
\end{tabular}

Tableau 10

Erreurs de déphasage $\phi_{\mathrm{V}}$ et incertitudes types associées en fonction de la fréquence $f$ du signal.

\begin{tabular}{|c|c|c|}
\hline$f(\mathrm{~Hz})$ & $\phi_{\mathrm{v}}(\mu \mathrm{rad})$ & $\sigma_{\phi \mathrm{v}}(\mu \mathrm{rad})$ \\
\hline 50 & 4 & 1 \\
\hline 100 & 9 & 1 \\
\hline 200 & 16 & 3 \\
\hline 400 & 31 & 4 \\
\hline
\end{tabular}

Tableau 11

Bilan d'incertitudes sur la mesure de la puissance active $P$ pour $60 \mathrm{~V} \leq U \leq 900 \mathrm{~V}, I, F$ et $\cos \Phi$ variables.

\begin{tabular}{|c|c|c|c|c|}
\hline \multicolumn{2}{|c|}{ Incertitude type relative } & \multicolumn{3}{|c|}{$\cos \Phi$} \\
\hline$I(\mathrm{~A})$ & $F(\mathrm{HZ})$ & 1 & 0,5 & 0,01 \\
\hline $0,1 \mathrm{~A}<I<10 \mathrm{~A}$ & 50 & 12 & 8 & 6 \\
\hline $10 \mathrm{~A} \leq I \leq 100 \mathrm{~A}$ & 50 & 23 & 17 & 15 \\
\hline $0,1 \mathrm{~A}<I<10 \mathrm{~A}$ & 400 & 15 & 12 & 10 \\
\hline $10 \mathrm{~A} \leq I \leq 100 \mathrm{~A}$ & 400 & 22 & 37 & 41 \\
\hline
\end{tabular}

Tableau 12

Bilan d'incertitudes sur la mesure de la puissance réactive $Q$ pour $60 \mathrm{~V} \leq U \leq 900 \mathrm{~V}, I, F$ et $\sin \Phi$ variables.

\begin{tabular}{|c|c|c|c|c|}
\hline \multicolumn{2}{|c|}{ Incertitude type relative } & \multicolumn{3}{c|}{$\sin \Phi$} \\
\hline$I(\mathrm{~A})$ & $F(\mathrm{HZ})$ & 1 & 0,5 & 0,001 \\
\hline $0,1 \mathrm{~A}<I \leq 10 \mathrm{~A}$ & 50 & 6 & 11 & 12 \\
\hline $10 \mathrm{~A}<I \leq 100 \mathrm{~A}$ & 50 & 15 & 21 & 23 \\
\hline $0,1 \mathrm{~A}<I \leq 10 \mathrm{~A}$ & 400 & 10 & 11 & 15 \\
\hline $10 \mathrm{~A}<I \leq 100 \mathrm{~A}$ & 400 & 41 & 28 & 22 \\
\hline
\end{tabular}

- les erreurs de déphasage $\phi_{\mathrm{V}}$ introduites par les deux voltmètres ont été déterminées expérimentalement [5]. Les résultats sont présentés dans le tableau 10.

\subsection{Bilans d'incertitudes}

Des bilans d'incertitudes détaillés sur les mesures des puissances active et réactive sont présentés dans les tableaux 11 et 12, à $53 \mathrm{~Hz}$ et $400 \mathrm{~Hz}$.

\subsection{Validation des mesures}

Les mesures de la puissance active ont déjà été validées [6] à $53 \mathrm{~Hz}$. La comparaison porte donc sur les mesures de la puissance réactive $Q$. Elle est réalisée, à $53 \mathrm{~Hz}$, en appliquant simultanément les signaux de tension et de
Tableau 13

Résultats de la comparaison sur la mesure de la puissance réactive monophasée à $53 \mathrm{~Hz}$. (les différentes voies d'entrée 1 , 2 et 3 du wattmàtre commercial sont testées indépendemment. Les calibres de $U$ et $I$ choisis correspondent aux valeurs nominales de $U$ et $I$ appliquées. Le wattmètre est utilisé en mode de mesure 4 fils-réactif.)

\begin{tabular}{|c|c|c|c|c|}
\hline$U(\mathrm{~V})$ & $I(\mathrm{~A})$ & $\phi\left(^{\circ}\right)$ & voie & $\varepsilon(\mathrm{ppm})$ \\
\hline 240 & 5 & $-30^{\circ}$ & 1 & -2 \\
& & $+30^{\circ}$ & 1 & 5 \\
& & $+90^{\circ}$ & 1 & 4 \\
\hline 240 & 5 & $-30^{\circ}$ & 2 & 2 \\
& & $+30^{\circ}$ & 2 & 9 \\
& & $+90^{\circ}$ & 2 & 9 \\
\hline 240 & 5 & $-30^{\circ}$ & 3 & 6 \\
& & $+30^{\circ}$ & 3 & 6 \\
& & $+90^{\circ}$ & 3 & 3 \\
\hline 60 & 5 & $-30^{\circ}$ & 1 & -15 \\
& & $+30^{\circ}$ & 1 & 4 \\
& & $+90^{\circ}$ & 1 & 17 \\
\hline 120 & 5 & $-30^{\circ}$ & 1 & -11 \\
& & $+30^{\circ}$ & 1 & -3 \\
& & $+90^{\circ}$ & 1 & 8 \\
\hline 480 & 5 & $-30^{\circ}$ & 1 & 4 \\
& & $+30^{\circ}$ & 1 & 29 \\
& & $+90^{\circ}$ & 1 & 18 \\
\hline
\end{tabular}

courant sur le wattmètre numérique à échantillonnage et un wattmètre commercial triphasé (utilisé uniquement en monophasé). Ce dernier a été préalablement étalonné à l'institut PTB avec une incertitude type relative (par rapport à la puissance apparente) de $20 \times 10^{-6}$ à $30 \times 10^{-6}$, pour des tensions comprises entre $60 \mathrm{~V}$ et $480 \mathrm{~V}$, des courants variant de $50 \mathrm{~mA}$ à $160 \mathrm{~A}$ et quel que soit le facteur de puissance.

Les résultats de la comparaison sont présentés dans le tableau 13. La grandeur $\varepsilon$ est définie par:

$$
\varepsilon=\frac{Q-Q_{\mathrm{PTB}}}{S}
$$

où $Q_{\mathrm{PTB}}$ et $Q$ sont les puissances réactives mesurées respectivement par le wattmètre étalon du PTB et le wattmètre numérique du LNE.

Il apparaît que, quelles que soient les conditions expérimentales, la valeur de $\varepsilon$ est toujours inférieure aux incertitudes de mesures des deux dispositifs.

\section{Conclusion}

Les domaines d'utilisation de l'étalon primaire des puissances électriques ont été élargis. Ainsi, l'étalon, basé sur la numérisation des signaux de tension $(60 \mathrm{~V} \leq$ $U \leq 900 \mathrm{~V})$ et de courant $(0,05 \mathrm{~A} \leq I \leq 100 \mathrm{~A})$, permet désormais de mesurer les puissances apparente $S$, active $P$ et réactive $Q$ dans le domaine des fréquences industrielles (de $45 \mathrm{~Hz}$ à $400 \mathrm{~Hz}$ ). Les mesures sont réalisées en régime sinusoïdal ou pour des signaux présentant un taux de distorsion harmonique inférieur à $0,1 \%$.

Pour valider le bon fonctionnement de cet étalon, une comparaison indirecte a été réalisée avec l'institut PTB 
par l'intermédiaire d'un wattmètre commercial triphasé. Les résultats montrent un très bon accord sur les mesures de $S, P$ et $Q$ à $53 \mathrm{~Hz}$. À ce jour, aucune comparaison n'a été réalisée à $400 \mathrm{~Hz}$ et, de ce fait, les mesures n'ont pu être validées à cette fréquence.

\section{Références}

[1] Espel P., PoletaefF A. et Bounouh A., "Static characterization of analog-to-digital converter", XIX IMEKO Fundamental and Applied Metrology, 6-11 septembre 2009, Lisbonne (Portugal).

[2] Espel P., Poletaeff A. et Bounouh A., "Characterization of analog-to-digital converters of a commercial

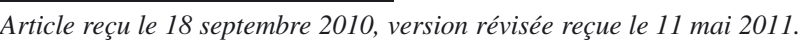

digital voltmeter in the $20 \mathrm{~Hz}$ to $400 \mathrm{~Hz}$ frequency range", 2009, Metrologia, 46, 578-584.

[3] IHLENFELD W.G.K. et al., "Characterisation of a high resolution analog to digital converter with a Josephson AC source", 2005, IEEE Trans. Instr. Meas., 54, 2, 649-652.

[4] Ihlenfield W.G.K., Mohns E., BAChMair H., RAMm G. et Moser H., "Evaluation of the synchronous generation and sampling technique", IEEE Trans. Instr. Meas., 52, 2, 371-374, 2003.

[5] EsPel P. et SVEnsson S., "Phase angle errors applying dual channel sampling", VI SEMETRO, Rio de Janeiro (Brésil), 21-23 septembre 2005, 45-48.

[6] EsPel P., « Développement d'un wattmètre numérique à échantillonnage. », Revue française de métrologie, 12, 2007, 3-12. 